\title{
APPENDIX
}

\section{Quality Management Tools and Approaches Matrix}

\begin{tabular}{|c|c|c|c|}
\hline \# & CASE STUDY NAME & TOOLS/APPROACH & TYPE \\
\hline 1 & A Summer Internship Journal & Checklist & Process Improvement \\
\hline 2 & Claims Payment Processing & Process Map & Process Improvement \\
\hline 3 & $\begin{array}{l}\text { Return-to-Work at a Home } \\
\text { Healthcare Agency }\end{array}$ & Swimlane Diagram & Process Improvement \\
\hline 4 & $\begin{array}{l}\text { The Ophthalmologist Who } \\
\text { Could Not See (The Waste) }\end{array}$ & Value Stream Map & Process Improvement \\
\hline 5 & Building a New IR Suite & Spaghetti Diagram & Process Improvement \\
\hline 6 & Emergency Department Heroes & Kaizen & Process Improvement \\
\hline 7 & $\begin{array}{l}\text { Hurricane Mia Hits the Patient } \\
\text { Access Call Center }\end{array}$ & $2 \times 2$ Matrix & Patient Experience \\
\hline 8 & $\begin{array}{l}\text { The Cowboy Doctor's Patient } \\
\text { Experience }\end{array}$ & Pareto Chart & Patient Experience \\
\hline 9 & $\begin{array}{l}\text { Patient Navigation at the } \\
\text { Orthopedic Clinic }\end{array}$ & Standardized Work & Patient Experience \\
\hline 10 & $\begin{array}{l}\text { HCAHPS and the Quiet-at-Night } \\
\text { Measure }\end{array}$ & Secret Shopper & Patient Experience \\
\hline 11 & $\begin{array}{l}\text { Discharge Phone Calls } \\
\text { (En Español) }\end{array}$ & Control Chart & Patient Experience \\
\hline 12 & $\begin{array}{l}\text { Patient Experience in Home } \\
\text { Care }\end{array}$ & $\begin{array}{l}\text { Managing for Daily } \\
\text { Improvement }\end{array}$ & Patient Experience \\
\hline
\end{tabular}




\begin{tabular}{|c|c|c|c|}
\hline$\#$ & CASE STUDY NAME & TOOLS/APPROACH & TYPE \\
\hline 13 & $\begin{array}{l}\text { Reducing Patient Falls: } \\
\text { The Sleuth Resident }\end{array}$ & Scatter Plot & Patient Safety \\
\hline 14 & Sustaining Hand Hygiene & The 5-Why? Method & Patient Safety \\
\hline 15 & $\begin{array}{l}\text { A Warning Letter From the State } \\
\text { Regulator }\end{array}$ & Poka-Yoke & Patient Safety \\
\hline 16 & Failure-to-Rescue & Escalation & Patient Safety \\
\hline 17 & CLIF's Medication Errors & Risk Mitigation Tool & Patient Safety \\
\hline 18 & A Mom's Story of Sepsis & $\begin{array}{l}\text { Failure Mode and Effects } \\
\text { Analysis }\end{array}$ & Patient Safety \\
\hline 19 & Operating Room Recovery & Data Analytics & Performance Improvement \\
\hline 20 & $\begin{array}{l}\text { Opioid Overdoses in the } \\
\text { Emergency Department }\end{array}$ & Fishbone Diagram & Performance Improvement \\
\hline 21 & $\begin{array}{l}\text { The Lunchroom: Physician } \\
\text { Engagement at a PCMH }\end{array}$ & Stakeholder Analysis & Performance Improvement \\
\hline 22 & The Fulfillment Affair & Gemba & Performance Improvement \\
\hline 23 & Composite Quality Score & Composite Score & Performance Improvement \\
\hline 24 & $\begin{array}{l}\text { Both/And Thinking in } \\
\text { Readmission Prevention }\end{array}$ & Performance Dashboard & Performance Improvement \\
\hline 25 & $\begin{array}{l}\text { Community Collaboration for } \\
\text { Suicide Prevention }\end{array}$ & Bar Charts and Histograms & Performance Improvement \\
\hline
\end{tabular}

HCAHPS, Hospital Consumer Assessment of Healthcare Providers and Systems; PCMH, patient centered medical home. 